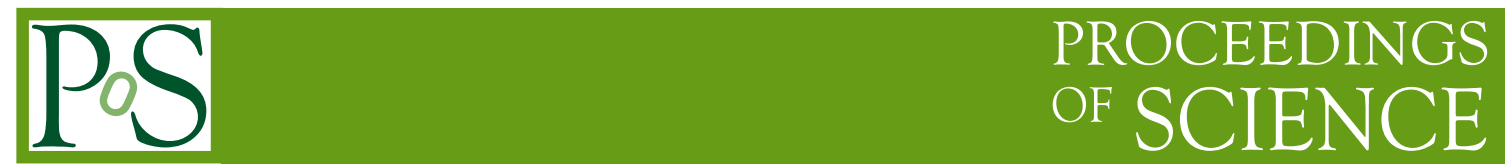

\title{
Low-energy hadron physics at KLOE/KLOE-2
}

\section{Xiaolin Kang ${ }^{* \dagger}$}

INFN-Laboratori Nazionali di Frascati

Via E. Fermi 40, 00044 Frascati (RM), Italy

E-mail: xiaolin.kangelnf.infn.it

The KLOE-2 experiment has completed its data-taking campaign at the $e^{+} e^{-}$DAФNE collider in Frascati, achieving the integrated luminosity goal of more than $5 \mathrm{fb}^{-1}$ at the $\phi$-meson peak. This new data sample, together with the KLOE data, corresponds to $2.4 \times 10^{10} \phi$ and $3.1 \times 10^{8} \eta$ meson decays, which supports plenty of precision measurements. This report presents the recent results and status of the low-energy hadron physics from KLOE/KLOE-2 experiments, including the search for the $P$ - and $C P$-violating process $\eta \rightarrow \pi^{+} \pi^{-}$obtaining the most stringent upper limit on the branching fraction, the study of the ChPT golden mode $\eta \rightarrow \gamma \gamma \pi^{0}$, the hypothetical $B$-boson searches in the decays $\phi \rightarrow \eta B$ and $\phi \rightarrow \eta(B \gamma) \gamma$ with $B \rightarrow \pi^{0} \gamma$, and the $\gamma \gamma$ interactions at KLOE-2 with the new installed taggers.

European Physical Society Conference on High Energy Physics - EPS-HEP2019 -

10-17 July, 2019

Ghent, Belgium

\footnotetext{
* Speaker.

${ }^{\dagger}$ On behalf of KLOE-2 Collaboration.
} 


\section{Introduction}

The KLOE-2 experiment, operated at the $e^{+} e^{-}$collider DAФNE, is a continuation of the KLOE [1] with an updated detector and an extended physics program, which includes the studies of light meson properties and decay dynamics with unprecedented statistics, tests of fundamental symmetries, and searches for phenomena beyond the Standard Model (SM). KLOE is a multipurpose detector, composed of a large cylindrical drift chamber (DC) [2] and an electromagnetic calorimeter (EMC) [3] surrounded by a superconducting coil providing a $0.52 \mathrm{~T}$ axial magnetic field. KLOE-2 additionally featured a cylindrical triple-GEM inner tracker $[4,5]$ and two calorimeters [6] close to the interaction region (IP) to improve vertex reconstruction near IP and increase the acceptance of the detector. Moreover, two couples of energy taggers [7] have been installed along the machine layout to study $\gamma \gamma$ fusion.

In March 2018 the KLOE-2 experiment has completed its data-taking campaign at the $e^{+} e^{-}$ DAФNE collider in Frascati, achieving the integrated luminosity goal of more than $5 \mathrm{fb}^{-1}$ at the $\phi$-meson peak. Together with the KLOE data, a total data sample of $8 \mathrm{fb}^{-1}$ has been collected, corresponding to $2.4 \times 10^{10} \phi$ and $3.1 \times 10^{8} \eta$ meson decays, which are invaluable to perform precise measurements and study low-energy hadron physics.

\section{Search for $\eta \rightarrow \pi^{+} \pi^{-}$}

The decay $\eta \rightarrow \pi^{+} \pi^{-}$violates both parity $(P)$ and charge conjugation parity $(C P)$ symmetry. In the $\mathrm{SM}$, this decay can proceed only through $C P$-violating weak interaction via a virtual $K_{S}^{0}$ meson and has branching fraction $\mathscr{B}\left(\eta \rightarrow \pi^{+} \pi^{-}\right) \leq 2 \times 10^{-27}[8,9]$. By introducing the possible contribution of the $C P$-violating QCD $\theta$-term to this decay, this upper limit can reach $10^{-17}[8,9]$. Allowing a $C P$-violation in the extended Higgs sector gives a slightly larger upper limit, $10^{-15}[8$, 9]. Therefore, any detection of a larger branching fraction would indicate a new source of $C P$ violation in the strong interaction.

With the first $350 \mathrm{pb}^{-1}$ data collected at $\phi$ resonance peak, KLOE collaboration set the best upper limit on the branching fraction, $\mathscr{B}\left(\eta \rightarrow \pi^{+} \pi^{-}\right) \leq 1.3 \times 10^{-5}$ at $90 \%$ confidence level [10]. A new preliminary limit has been determined by the KLOE- 2 collaboration with an integrated luminosity of $1.6 \mathrm{fb}^{-1}$.

The selection of $\phi \rightarrow \eta \gamma, \eta \rightarrow \pi^{+} \pi^{-}$decays requires: i) one vertex reconstructed in the tracking detector with two opposite-charge tracks reaching the calorimeter and required to be at large polar angle $45^{\circ}<\theta<135^{\circ}$ and ii) one prompt photon reconstructed by an energy cluster in EMC not associated to any track. The timing requirement $\left|T_{c l}-R_{c l} / c\right|<\min \left(5 \sigma_{t}\left(E_{c l}\right), 2 n s\right)$ is used to suppress electronic noise and energy requirement $250 \leq E_{c l} \leq 470 \mathrm{MeV}$ to suppress initial state radiation background, where $T_{c l}$ is photon time, $R_{c l}$ is the distance from the IP, $\sigma_{t}$ is the energydependent time resolution, and $E_{c l}$ is photon energy. The angle $\psi$ between the missing momentum of the di-pion system and the selected photon direction has to be less than $0.05 \mathrm{rad}$ to reject the background from $\phi \rightarrow \rho \pi \rightarrow \pi^{+} \pi^{-} \pi^{0}$ decays. Main background contamination originates from $e^{+} e^{-} \rightarrow(\gamma) \gamma \pi^{+} \pi^{-},(\gamma) \gamma e^{+} e^{-},(\gamma) \gamma \mu^{+} \mu^{-}$and $\phi \rightarrow \rho \pi \rightarrow \pi^{+} \pi^{-} \pi^{0}$ with a lost photon decays. The time-of-flight techniques are use to separate $\gamma e^{+} e^{-}$from the selected $\gamma \pi^{+} \pi^{-}$events. While $\gamma \mu^{+} \mu^{-}$ and the remain $\phi \rightarrow \rho \pi \rightarrow \pi^{+} \pi^{-} \pi^{0}$ events can be rejected using the track-mass variable, computed 
assuming the $\phi$-meson decays to two particles of identical mass and one photon, as shown in Fig. 1 left panel.

The candidates surviving the above selection are mainly $(\gamma) \gamma \pi^{+} \pi^{-}$events. The $\pi^{+} \pi^{-}$mass spectrum, $M\left(\pi^{+} \pi^{-}\right)$, is used to look for the presence of $\eta$ resonance, shown in the right panel of Fig. 1. No peak is observed in the distribution of $M\left(\pi^{+} \pi^{-}\right)$in the vicinity of $m_{\eta}$. The upper limit on the number of signal events is extracted by using a Bayesian approach. The irreducible background is evaluated by performing a fit to the observed $M\left(\pi^{+} \pi^{-}\right)$with a third-order polynomial function while the $\eta \rightarrow \pi^{+} \pi^{-}$signal is described with the Monte Carlo (MC) simulated shape. The preliminary upper limit on the branching fraction is determined to be $5.8 \times 10^{-6}$ at $90 \%$ confidence level. With all the KLOE and KLOE-2 data the upper limit is expected to reach $2.7 \times 10^{-6}$.
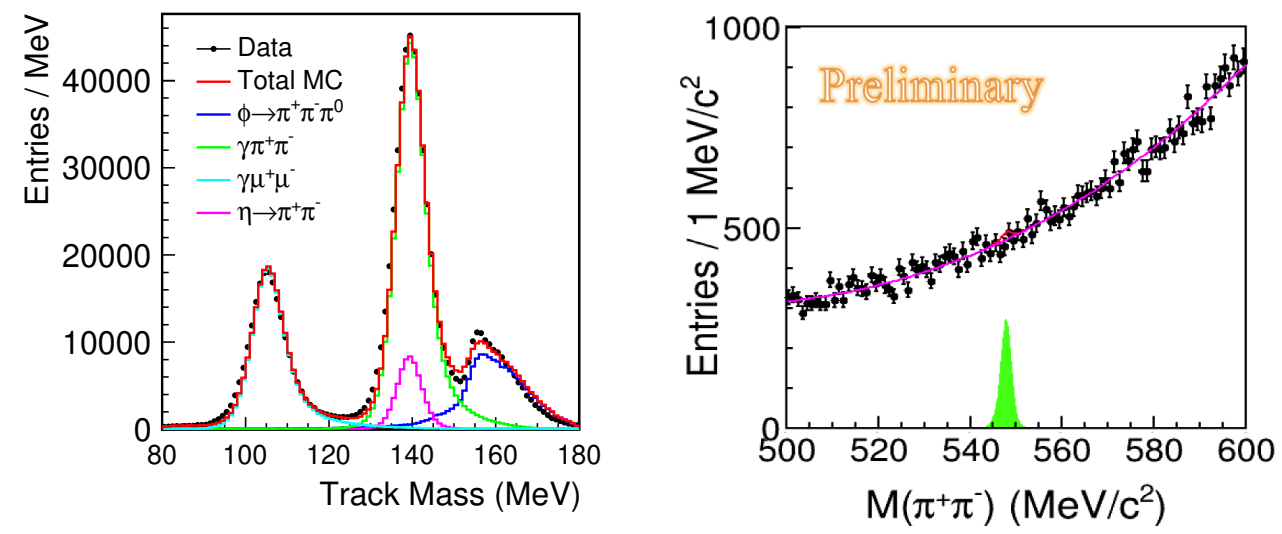

Figure 1: Left: track mass distributions. The black dots are data; the red histogram is total MC contributions in the hypothesis of no $\eta \rightarrow \pi^{+} \pi^{-}$signal; the blue histogram is $\phi \rightarrow \rho \pi$ with $\rho \rightarrow \gamma \pi$ or $\pi \pi$ estimated from MC simulation; the green histogram is $e^{+} e^{-} \rightarrow(\gamma) \gamma \pi^{+} \pi^{-}$; the cyan histogram is $e^{+} e^{-} \rightarrow \gamma \mu^{+} \mu^{-}$; while the expected signal is shown in violet. Right: $\pi^{+} \pi^{-}$invariant mass distribution. The black dots with error bar are data; the solid curve is a third-order polynomial function with superimposed a MC signal shape, shown as the shaded histogram in arbitrary units.

\section{The $\eta \rightarrow \pi^{0} \gamma \gamma$ decay}

The doubly radiative decay $\eta \rightarrow \pi^{0} \gamma \gamma$ decay is an important test of Chiral Perturbation Theroy (ChPT) because of its sensitivity to the $p^{6}$ term on both the branching fraction and the $M(\gamma \gamma)$ spectrum. The contribution from $\mathscr{O}\left(p^{2}\right)$ is null for neutral mesons and $\mathscr{O}\left(p^{4}\right)$ highly suppressed by G parity [11]. The PDG [12] branching fraction $\mathscr{B}\left(\eta \rightarrow \gamma \gamma \pi^{0}\right)=(25.2 \pm 2.5) \times 10^{-5}$ comes from the Crystal Ball measurements [13,14]. A preliminary KLOE measurement, based on $450 \mathrm{pb}^{-1}$ data, is $\mathscr{B}\left(\eta \rightarrow \gamma \gamma \pi^{0}\right)=\left(8.4 \pm 2.7_{\text {stat }} \pm 1.4_{\text {syst }}\right) \times 10^{-5}[15]$, which is significantly lower than the Crystall Ball and two standard deviations lower than the predictions from Chiral-loop, L $\sigma \mathrm{M}$ and VMD $[11,16]$.

Reanalysis for this decay with four times larger data sample is in progress at KLOE-2. The experimental challenges of measuring this decay are the smallness of the rate for doubly radiative 
decays and the large background, in particular from $\eta \rightarrow 3 \pi^{0} \rightarrow 6 \gamma$ decays with lost or merged photons. In addition, backgrounds from $\phi \rightarrow a_{0}\left(\eta \pi^{0}\right) \gamma, f_{0}\left(\pi^{0} \pi^{0}\right) \gamma, e^{+} e^{-} \rightarrow \omega\left(\gamma \pi^{0}\right) \pi^{0}$ also mimic the signal.

To suppress backgrounds, kinematic fits under different signal or background hypotheses are performed by enforcing the energy-momentum conservation and constraints on the time-of-flight of neutral clusters. Multi-variable analysis with cluster shape information is also performed to separate single photon from merged photon clusters. The preliminary invariant mass distribuion of $\pi^{0} \gamma \gamma$ is shown in Fig. 2. The signal-to-background ratio amounts to 0.4 with a global selection efficiency $21 \%$ determined from the MC simulation. Further background subtraction and signal extraction is in progress and the preliminary result on the branching fraction is forthcoming. This decay also provides a probability of finding a leptophobic mediator $B$-boson between the dark sector and the SM particles via the decay chain $\eta \rightarrow \gamma B, B \rightarrow \gamma \pi^{0}$ [17].

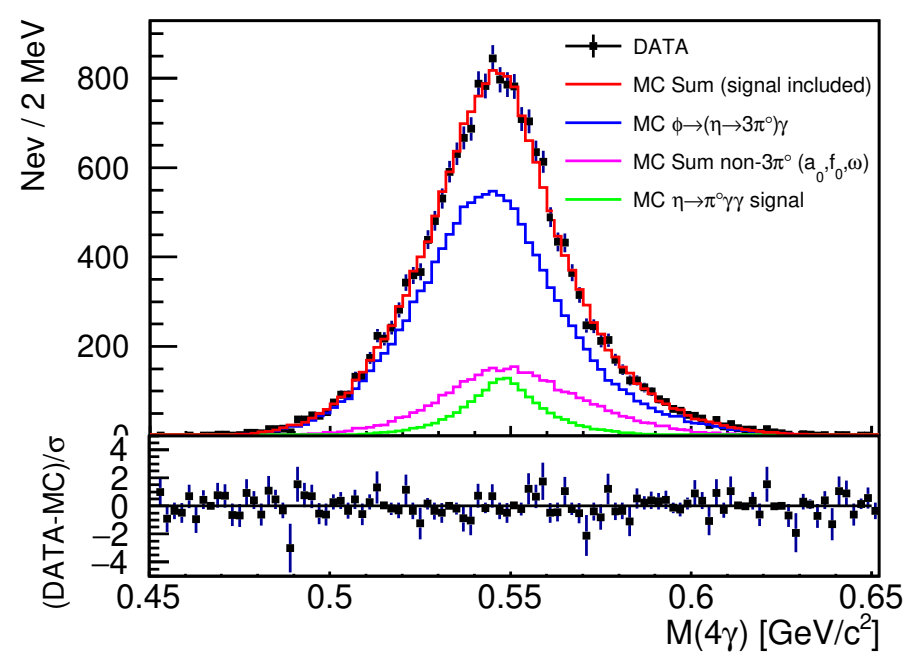

Figure 2: Upper: invariant mass spectrum of $\pi^{0} \gamma \gamma$ candidates. The black dots with error bars are data; the red histogram is the sum of MC background contributions; the blue histogram is $\eta \rightarrow 3 \pi^{0}$, the violet histogram is the sum of the backgrounds from $\phi \rightarrow \gamma a_{0}\left(\eta \pi^{0}\right), \gamma f_{0}\left(\pi^{0} \pi^{0}\right)$, and $e^{+} e^{-} \rightarrow \omega\left(\gamma \pi^{0}\right) \pi^{0}$; the expected signal is shown in green. Lower panel shows the ratio between data and total MC simulation.

\section{Search for $B$-boson}

The model presented in Ref [17] proposes a gauge boson, dubbed $B$, that couples to the baryon number and arises from a $U(1)_{B}$ gauge symmetry in the GeV-scale, especially in the range 100 $\mathrm{MeV}<m_{B}<1 \mathrm{GeV}$. The interaction Lagrangian is:

$$
\mathscr{L}=\frac{g_{B}}{3} \bar{q} \gamma^{\mu} q B_{\mu},
$$

where $B_{\mu}$ is the new gauge field coupling to the baryon number. The gauge coupling $g_{B}$ is universal for all quarks $q$, estimated to be $g_{B} \leq 10^{-2} \times\left(m_{B} / 100 \mathrm{MeV}\right)$ [17]. With quantum number $I^{G}\left(J^{P C}\right)=0^{-}\left(1^{--}\right)$, the $B$-boson decays in a similar way as the $\omega$-meson. 
In KLOE the searches for $B$-boson with mass below $600 \mathrm{MeV}$ are performed, where the dominant decay is $B \rightarrow \pi^{0} \gamma$. Except the decay channel $\phi \rightarrow \eta(B \gamma) \gamma$ with $B \rightarrow \pi^{0} \gamma$, mentioned in section 3, the decay $\phi \rightarrow \eta B\left(\pi^{0} \gamma\right)$ is also used to search for a $B$-boson. The SM decay $\phi \rightarrow a_{0}(980)\left(\eta \pi^{0}\right) \gamma$ with same final states and $\phi \rightarrow \eta\left(3 \pi^{0}\right) \gamma$ with lost or merged photons strongly mimic the possible signal. To suppress backgrounds, kinematic fits under different signal or background hypotheses are performed by enforcing the energy-momentum conservation, constraints on the time-of-flight of neutral clusters and the invariant masses of $\gamma \gamma$ pairs to the nominal $\eta$ and $\pi^{0}$ masses.

In figure 3, preliminary invariant mass spectra of $\pi^{0} \gamma$ from the selected $\eta \pi^{0} \gamma$ candidates are shown. In case of formation of the $B$-boson, the signature is an enhancement in the corresponding $\pi^{0} \gamma$ invariant mass. In case no signal is observed an upper limit in the coupling of such a dark matter mediator to the SM as a function of its mass will be established.

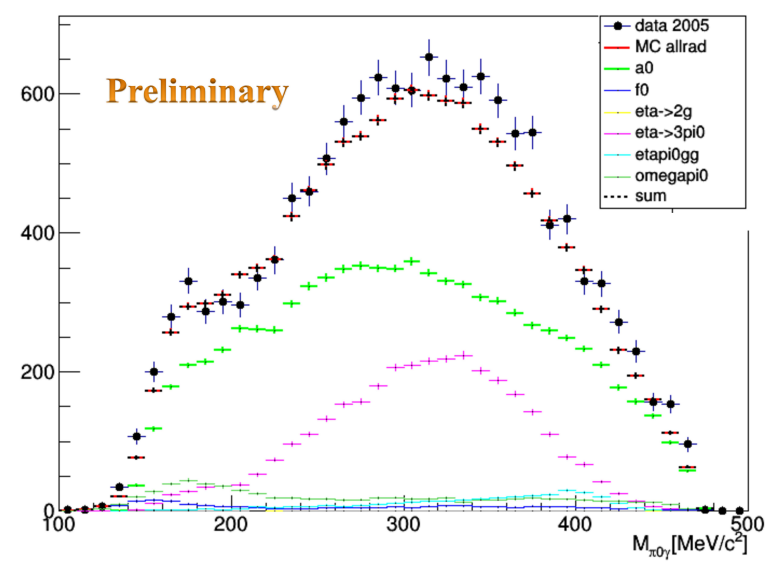

Figure 3: Invariant mass spectrum of $\pi^{0} \gamma$ candidates for the channel $\phi \rightarrow \eta B \rightarrow \eta \pi^{0} \gamma$. The black dots with error bars are data; the red dots are the sum of MC simulated background contributions; the main backgrounds are $\phi \rightarrow a_{0}(980)\left(\eta \pi^{0}\right) \gamma$ (green), $\phi \rightarrow \eta\left(3 \pi^{0}\right) \gamma$ (violet), $\phi \rightarrow f_{0}(980)\left(\pi^{0} \pi^{0}\right) \gamma$ (blue), $\phi \rightarrow$ $\eta(\gamma \gamma) \gamma$ (yellow), $\phi \rightarrow \eta\left(\pi^{0} \gamma \gamma\right) \gamma$ (cyan), $\phi \rightarrow \omega\left(\pi^{0} \gamma\right) \pi^{0}$ (light green).

\section{5. $\pi^{0}$ production from $\gamma \gamma$ scattering}

$\gamma \gamma$ couplings to mesons and the partial widths provide information about their structure and can be measured in the $e^{+} e^{-} \rightarrow e^{+} e^{-} \gamma^{*} \gamma^{*} \rightarrow e^{+} e^{-} X$ processes, where $X$ is a symbol refering to the final state with $J^{P C}=0^{ \pm+}, 2^{ \pm+}$final state. It can be $\pi^{0}, \pi \pi, \eta \pi$, or $\eta$ [18]. In case of a single particle $R$ produced in the final state, the cross section for the decays is proportional to the square of transition form factor:

$$
\sigma_{\gamma \gamma \rightarrow R\left(q_{1}, q_{2}\right)} \propto \Gamma_{R \rightarrow \gamma \gamma} \frac{8 \pi^{2}}{M_{R}} \delta\left(\left(q_{1}+q_{2}\right)^{2}-M_{R}^{2}\right)\left|F\left(q_{1}^{2}, q_{2}^{2}\right)\right|^{2} .
$$

Therefore, a precise knowledge of the $\gamma \gamma$ decay width provides a measurement of the transition form factor, which contributes to the hadronic light-by-light term of the anomalous magnetic moment of the muon [19]. 
The most precise measurement $\Gamma_{\pi^{0} \rightarrow \gamma \gamma}=7.82 \pm 0.22 \mathrm{eV}$, is obtained from the Primakoff effect, reaching an accuracy of 2.8\% [20]. A recent report in the PhiPsi2019 conference from the same Collaboration presented a more precision result $\Gamma_{\pi^{0} \rightarrow \gamma \gamma}=7.802 \pm 0.117 \mathrm{eV}$ [21].

Two High Energy Tagger (HET) stations, installed in KLOE-2 to tag electrons and positrons from the reaction $e^{+} e^{-} \rightarrow e^{+} e^{-} \gamma^{*} \gamma \rightarrow e^{+} e^{-} \pi^{0}$, will give the opportunity to investigate $\gamma^{*} \gamma \rightarrow \pi^{0}$ physics at the $\phi$ resonance, aiming to achieve an accuracy of $\mathscr{O}(1 \%)$ matching the current theory precision and the first measurement of the transition form factor $F_{\pi^{0} \gamma^{*} \gamma}\left(q^{2}\right)$ in the space-like region at low momentum transfer $q_{\gamma^{*}}^{2} \leq 0.1 \mathrm{GeV}^{2}$ [19].

The HET detectors have been installed in roman pots just at the exit of the DA $\Phi$ NE dipole magnets, $11 \mathrm{~m}$ away from the IP, both at the positron and electron sides. The HET sensitive area is made of a set of 28 plastic scintillators. The measured time resolution of $550 \mathrm{ps}$ gives the possibility to clearly identify the correct bunch crossing $\left(\Delta T_{\text {bunch }} \sim 2.7 \mathrm{~ns}\right)$. The counting rate is dominated by low-angle radiative Bhabha scattering events and their effective cross section as a function of the data-taking period has been used to determine the detector acceptance $\times$ efficiency and for data quality purposes. An effective cross section of the order of $10 \mathrm{mb}$ has been measured, with big instabilities for the scintillators closest to the beam.

For the $\pi^{0}$ search a sub-set of HET plastic scintillators has been used showing operational stability over a time scale of years. Candidates of single- $\pi^{0}$ production from $\gamma \gamma$ scattering have been pre-filtered recording information on the hit in the tagger, trigger, DAФNE operational parameters, clusters and tracks reconstructed in the KLOE detector. The signal events are required to have hits in HET station and at least one bunch in KLOE spectrometer assoicated with only 2 clusters in the KLOE EMC, in a given time window around the trigger signal. Very loose kinematics cuts are applied to reconstructed data. The HET-KLOE coincidence data sample is dominated by time coincidences from independent events (so called accidentals), even at one-bunch-crossing level. Since the HET data acquisition window is about 2.5 times larger than that of the main part of the spectrometer, the sub-sample out-of-time window of the two detectors is continuously acquired at the same time of the coincidence sample and thus it can be correctly subtracted.

By analysing a sample of $500 \mathrm{pb}^{-1}$, a clear statistical evidence of correlated coincidence events between the tagger station hits and KLOE calorimeter clusters has been observed for the first time, after an event-by-event subtraction of the registered accidentals. Multi-variable analysis is used to seperate signal from the radiative Bhabha background. Further identification of background events and analysis with more statistics is ongoing in order to study in more detail the tagged sample.

\section{Conclusions}

The unique worldwide sample collected by the KLOE/KLOE-2 experiments are used to perform precise measurements in low-energy hadron physics and search for physics beyond SM. A preliminary and most stringent upper limit for the $P$ - and $C P$-violating decay $\eta \rightarrow \pi^{+} \pi^{-}$has been obtained, which will supersede the previous result from KLOE Collaboration. A precise study of the ChPT golden mode $\eta \rightarrow \pi^{0} \gamma \gamma$ is in progress, which may shed light on the puzzle of its branching fraction. Searches for leptophobic dark matter mediator are being performed in two different processes. We also note a progress in the $\gamma^{*} \gamma^{*} \rightarrow \pi^{0}$ search using KLOE-2 data. 


\section{Acknowledgments}

We warmly thank our former KLOE colleagues for the access to the data collected during the KLOE data taking campaign. We thank the DAФNE team for their efforts in maintaining low background running conditions and their collaboration during all data taking. We want to thank our technical staff: G.F. Fortugno and F. Sborzacchi for their dedication in ensuring efficient operation of the KLOE computing facilities; $\mathrm{M}$. Anelli for his continuous attention to the gas system and detector safety; A. Balla, M. Gatta, G. Corradi and G. Papalino for electronics maintenance; C. Piscitelli for his help during major maintenance periods. This work was supported in part by the Polish National Science Centre through the Grants No. 2013/11/B/ST2/04245, 2014/14/E/ST2/00262, 2014/12/S/ST2/00459, 2016/21/N/ST2/01727, 2016/23/N/ST2/01293, 2017/26/M/ST2/00697.

\section{References}

[1] The KLOE Collaboration, The KLOE Detector Technical Proposal, LNF-93/002, 1993; S. Bertolucci (KLOE Collaboration), A Status Report of KLOE at DAФNE, in Proc. 19th International Symposium on Lepton and Photon Interactions at High Energies, Stanford, USA, 1999, hep-ex/0002030.

[2] M. Adinolfi et al., Nucl. Instr. Meth. A 488 (2002) 51.

[3] M. Adinolfi et al., Nucl. Instr. Meth. A 482 (2002) 364.

[4] G. Bencivenni, D. Domenici, Nucl. Instr. Meth. A 581 (2007) 221.

[5] A. Di Cicco, G. Morello, Acta Phys. Pol. B 46 (2015) 73.

[6] F. Happacher, M. Martini, Acta Phys. Pol. B 46 (2015) 87.

[7] D. Babusci et al., Acta Phys. Pol. B 46 (2015) 81.

[8] C. Jarlskog, E. Shabalin, Phys. Scr. T 99 (2002) 23.

[9] E. Shabalin, Phys. Scr. T 99 (2002) 104.

[10] F. Ambrosino et al. (KLOE Collaboration), Phys. Lett. B 606 (2005) 276.

[11] L. Ametller, J. Bijnens, A. Bramon and F. Cornet, Phys. Lett. B 276 (1992) 185.

[12] M. Tanabashi et al. (Particle Data Group), Phys. Rev. D 98 (2018) 030001.

[13] S. Prakhov et al. (Crystal Ball Collaboration at AGS), Phys. Rev. C 78 (2008) 015206.

[14] B. M. K. Nefkens et al. (A2 Collaboration at MAMI), Phys. Rev. C 90 (2014) 025206.

[15] B. Di Micco et al. (KLOE Collaboration), Acta Phys. Slov. 56 (2006) 403.

[16] R. Escribano, S. Gonzàlez-Solis, R. Jora and E. Royo, arXiv:1812.08454 [hep-ph].

[17] S. Tulin, Phys. Rev. D 89 (2014) 114008.

[18] G. Amelino-Camelia et al., Eur. Phys. J. C 68 (2010) 619.

[19] D. Babusci et al., Eur. Phys. J. C 72 (2012) 1917.

[20] I. Larin et al., Phys. Rev. Lett. 106 (2011) 162303.

[21] I. Larin, talk in PhiPsi2019, https://indico.inp.nsk.su/event/15/session/2/contribution/34/material/slides/0.pdf 